\title{
Snap-Through of Unsymmetric Laminates Using Piezocomposite Actuators
}

\author{
Marc R. Schultz \\ Michael W. Hyer ${ }^{2}$ \\ R. Brett Williams ${ }^{3}$ \\ W. Keats Wilkie ${ }^{1}$ \\ Daniel J. Inman ${ }^{4}$ \\ ${ }^{1}$ Structural Dynamics Branch, MS 230 \\ NASA Langley Research Center \\ Hampton, VA 23681-2199 \\ ${ }^{2}$ Department of Engineering Science and Mechanics (0219) \\ Virginia Polytechnic Institute and State University \\ Blacksburg, VA 24061 \\ hyerm@vt.edu, tel: 540-231-5372,fax: 540-231-4574 \\ ${ }^{3}$ NASA Jet Propulsion Laboratory \\ Pasadena, CA 91109-8099 \\ ${ }^{4}$ Center for Intelligent Material Systems and Structures \\ Department of Mechanical Engineering \\ Virginia Polytechnic Institute and State University \\ Blacksburg, VA 24061
}




\begin{abstract}
The paper discusses the concept of using a piezoceramic actuator bonded to one side of a twolayer unsymmetric cross-ply $[0 / 90]_{\mathrm{T}}$ laminate to provide the moments necessary to snap the laminate from one stable equilibrium shape to another. This concept could be applied to the morphing of structures. A model of this concept, which is based on the Rayleigh-Ritz technique and the use of energy and variational methods, is developed. The experimental phase of the study is discussed, including the measurement of the voltage level needed to snap the laminate. The voltage measurements and shapes are compared with predictions of the models and the agreement between measurements and the predictions are reasonable, both qualitatively and quantitatively. Suggestions for future activities are presented.
\end{abstract}

Keywords: MFC actuator, morphing, Rayleigh-Ritz technique, stability, multistable

\title{
INTRODUCTION
}

As is well known, a thin, unsymmetrically laminated, elevated temperature cure, fiber-reinforced composite laminate with no external loads, such as a two-layer cross-ply graphite-epoxy $[0 / 90]_{\mathrm{T}}$ laminate, can have multiple equilibrium shapes when cooled from the curing temperature to a lower operating temperature; that is, the laminate can be multistable. In the case of a cross-ply laminate, when the geometry is such that the laminate is multistable, there are two stable cylindrical shapes and one unstable saddle shape. The laminate can be changed from one stable cylindrical shape to the other by a simple snap-through action by applying moments along opposite edges of the laminate. The unstable shape, of course, is never achievable. This multistable phenomenon has been studied by a number of investigators [1-4], and is a result of the coupling of the residual stresses due to cooling with the geometric nonlinearities due to the large out-of-plane deflections involved.

This study considers the use of the NASA Langley Research Center Macro-Fiber Composite Actuator (MFC), an orthotropic piezoceramic actuator, to effect snap-through behavior of a $[0 / 90]_{\mathrm{T}}$ laminate; MFC actuators are prepackaged and the actuation is accomplished by applying a voltage to the actuator. The multistable characteristic could be applied to the design of morphing structures. The geometry of the structure that was considered in this study is shown schematically in fig. 1; the MFC actuator is centrally bonded to one side of the laminate to form the laminate-actuator combination. Predictions from a model of the laminate-actuator combination based on the Rayleigh-Ritz technique will be compared with experiments. In particular, predictions of the shapes of the laminate-actuator combination and of the voltage applied to the actuator that causes snap-through are compared with experiments. 


\section{THE MACRO-FIBER COMPOSITE ACTUATOR}

The MFC actuator consists of piezoceramic PZT macrofibers embedded in a structural epoxy matrix and sandwiched between interdigitally-electroded polyimide films [5]. Two-dimensional modeling of the piezoelectrically-induced dilatational strains of the MFC actuators used in this study can be accomplished in a manner similar to the typical modeling of a monolithic piezoelectric wafer, namely,

$$
\begin{aligned}
\varepsilon_{1}^{E} & =d_{11} \frac{\Delta V}{\Delta x_{1}} \\
\varepsilon_{2}^{E} & =d_{12} \frac{\Delta V}{\Delta x_{1}} \\
\gamma_{12}^{E} & =0
\end{aligned}
$$

where $d_{11}$ and $d_{12}$ are the effective piezoelectric constants for the MFC actuator, $\Delta V$ is the voltage applied to the MFC actuator, and $\Delta x_{1}$ is the electrode spacing. The term effective is used to describe the piezoelectric constants because the interdigitated electrodes create a nonuniform electric field in the actuator, but by using $\Delta V / \Delta x_{1}$, an average, or effective, electric field in the actuator is considered; as with properties of other composites, the electomechanical properties of the MFC actuator are "smeared" within the volume of the actuator. In the above strain notation, and throughout this paper, the principal material coordinate system nomenclature standard to fiber-reinforced composite materials is used. That is, the material coordinate system is denoted by the subscripts 1,2 , and 3 , and the global coordinate system is denoted by $x, y$, and $z$, as shown in fig. 1 .

\section{MODEL DEVELOPMENT}

The analytical model that was developed for this study was divided into three parts: cooling the cured laminate, bonding the actuator to the laminate, and applying voltage to the laminate-actuator combination. As in [2], in accordance with the requirements of the Rayleigh-Ritz technique, the current model relied on using good approximations of the displacement fields in conjunction with an extension of classical lamination theory by including geometric nonlinearities in the straindisplacement relations. Stationary values of the total potential energy of the laminate are sought. It was believed that a ten-parameter approach could be used for predicting the shapes of the laminateactuator combination. Accordingly, in each of the three steps, the three components of displacement of the geometric midsurface of the laminate, the reference surface in this analysis, were approximated by 


$$
\begin{aligned}
& u^{0}=c_{5} x+\frac{1}{3} c_{6} x^{3}+c_{7} x y^{2}-\frac{1}{6} c_{1}^{2} x^{3}-\frac{1}{8} c_{1} c_{3} x^{4}-\frac{1}{40} c_{3}^{2} x^{5} \\
& v^{0}=c_{8} y+c_{9} x^{2} y+\frac{1}{3} c_{10} y^{3}-\frac{1}{6} c_{2}^{2} y^{3}-\frac{1}{8} c_{2} d_{4} y^{4}-\frac{1}{40} c_{4}^{2} y^{5} \\
& w^{0}=\frac{1}{2}\left(c_{1} x^{2}+c_{2} y^{2}\right)+\frac{1}{6}\left(c_{3} x^{3}+c_{3} y^{3}\right)
\end{aligned}
$$

where $u^{0}, v^{0}$, and $w^{0}$ are the displacements in the $x$-, $y$-, and $z$-directions, respectively. The ten parameters $c_{1}$ through $c_{10}$ are unknown, but to-be-determined, coefficients. Because of symmetry requirements on the displacement, the third-order $w^{0}$-displacement polynomial could not be used to represent the $w^{0}$-displacement field over the entire laminate. However, a quarter-symmetry argument was used to allow the use of this polynomial to represent the $w^{0}$-displacement field, and the total potential energy was calculated over only one-quarter of the laminate. It should also be mentioned that, consistent with eq. 2, the laminate was considered to be fixed at the geometric center, and with no external applied loads.

\section{Step I: Laminate cooling from cure}

The first step of the model was to determine the initial cooled shape of the two-layer $[0 / 90]_{\mathrm{T}}$ laminate. Because this step would not include the MFC actuator, and no forces were applied to the laminate, only thermal effects within the laminate needed to be considered. Accordingly, the total potential energy, $\Pi$, used in Step I was the strain energy of the cooled laminate, $\Pi_{1}$, and is given by

$$
\Pi=\Pi_{1}=4\left(\frac{1}{2} \int_{0}^{\frac{L_{x}}{2}} \int_{0}^{\frac{L_{y}}{2}} \int_{z_{0}}^{z_{2}}\left[\left(\sigma_{x}-\sigma_{x}^{T}\right) \varepsilon_{x}+\left(\sigma_{y}-\sigma_{y}^{T}\right) \varepsilon_{y}+\left(\sigma_{x y}-\sigma_{x y}^{T}\right) \gamma_{x y}\right] d x d y d z\right)
$$

where the $\sigma \mathrm{s}$ are the stresses in the laminate, the $\sigma^{T}$ s are the so-called thermally-induced stresses, the $\varepsilon s$ and $\gamma_{\mathrm{xy}}$ are the strains, and $L_{x}$ and $L_{y}$ are the sidelengths of the laminate when flat as depicted in fig. 1. Also as shown in fig. 1 , the thickness coordinates $z_{0}$ and $z_{2}$ are the coordinates for the bottom of the $0^{\circ}$ graphite-epoxy layer and the top of the $90^{\circ}$ graphite-epoxy layer, respectively.

The stresses in eq. 3 are given by

$$
\begin{aligned}
& \sigma_{x}=\bar{Q}_{11} \varepsilon_{x}+\bar{Q}_{12} \varepsilon_{y}+\bar{Q}_{16} \gamma_{x y}-\sigma_{x}^{T} \\
& \sigma_{y}=\bar{Q}_{12} \varepsilon_{x}+\bar{Q}_{22} \varepsilon_{y}+\bar{Q}_{26} \gamma_{x y}-\sigma_{y}^{T} \\
& \sigma_{x y}=\bar{Q}_{16} \varepsilon_{x}+\bar{Q}_{26} \varepsilon_{y}+\bar{Q}_{66} \gamma_{x y}-\sigma_{x y}^{T}
\end{aligned}
$$

where the $\bar{Q}$ s are the transformed reduced stiffnesses of the graphite-epoxy layers and the thermallyinduced stresses are given by 


$$
\begin{aligned}
& \sigma_{x}^{T}=\bar{Q}_{11} \varepsilon_{x}^{T}+\bar{Q}_{12} \varepsilon_{y}^{T}+\bar{Q}_{16} \gamma_{x y}^{T} \\
& \sigma_{y}^{T}=\bar{Q}_{12} \varepsilon_{x}^{T}+\bar{Q}_{22} \varepsilon_{y}^{T}+\bar{Q}_{26} \gamma_{x y}^{T} \\
& \sigma_{x y}^{T}=\bar{Q}_{16} \varepsilon_{x}^{T}+\bar{Q}_{26} \varepsilon_{y}^{T}+\bar{Q}_{66} \gamma_{x y}^{T}
\end{aligned}
$$

with

$$
\begin{aligned}
\varepsilon_{x}^{T} & =\alpha_{x} \Delta T \\
\varepsilon_{y}^{T} & =\alpha_{y} \Delta T \\
\gamma_{x y}^{T} & =\alpha_{x y} \Delta T=0
\end{aligned}
$$

The $\alpha$ s are the coefficients of thermal deformation of the layer and $\Delta T$ is the temperature change from the cure temperature to room temperature, here the operating temperature. Note that $\bar{Q}_{16}, \bar{Q}_{26}$, and $\alpha_{x y}$ are retained in the formulation, even though they are zero for 0 and $90^{\circ}$ layers.

For small strains and moderate rotations, the midsurface strains and curvatures in the laminate are given by the von Kármán approximation to the more general strain-displacement relations, namely,

$$
\begin{array}{ll}
\varepsilon_{x}^{0}=\frac{\partial u^{0}}{\partial x}+\frac{1}{2}\left(\frac{\partial w^{0}}{\partial x}\right)^{2} & \kappa_{x}^{0}=-\frac{\partial^{2} w^{0}}{\partial x^{2}} \\
\varepsilon_{y}^{0}=\frac{\partial v^{0}}{\partial y}+\frac{1}{2}\left(\frac{\partial w^{0}}{\partial y}\right)^{2} & \kappa_{y}^{0}=-\frac{\partial^{2} w^{0}}{\partial y^{2}} \\
\gamma_{x y}^{0}=\frac{\partial u^{0}}{\partial y}+\frac{\partial v^{0}}{\partial x}+\frac{\partial w^{0}}{\partial x} \frac{\partial w^{0}}{\partial y} & \kappa_{x y}^{0}=-2 \frac{\partial^{2} w^{0}}{\partial x \partial y}
\end{array}
$$

According to the Kirchhoff hypothesis, the strains as a function of the thickness location, $z$, are given by

$$
\begin{aligned}
& \varepsilon_{x}=\varepsilon_{x}^{0}+z \kappa_{x}^{0} \\
& \varepsilon_{y}=\varepsilon_{y}^{0}+z \kappa_{y}^{0} \\
& \gamma_{x y}=\gamma_{x y}^{0}+z \kappa_{x y}^{0}
\end{aligned}
$$

By using eq. 2 in eqs. 7 and 8 and, in turn, substituting these results into eqs. 4 and 3, taking into account eqs. 5 and 6 , and carrying out the integrations of eq. 3 , the total potential energy is reduced to an algebraic equation in terms of material properties, geometry, and the coefficients $c_{1}$ through $c_{10}$. These coefficients are determined by solving the ten simultaneous nonlinear algebraic equations that result from equating to zero the first variation of total potential energy with respect to these coefficients, namely,

$$
\frac{\partial \Pi}{\partial c_{i}}=0 \quad i=1, n
$$


Stability of the solution is determined by checking the positive definiteness of the ten-by-ten matrix associated with the second variation of the total potential energy with respect to these ten coefficients. The algebraic manipulations, integrations, and differentiations in this and the following steps were all accomplished with the aid of Mathematica [6].

\section{Step II: Bonding the MFC actuator to the laminate}

The MFC actuator was to be bonded to the laminate by placing the laminate and actuator, with adhesive, inside a vacuum bag, and using a vacuum pump to evacuate the bag to pull the laminate and the actuator into contact until the adhesive cured. The result was that the contacting surfaces of the actuator and the laminate would develop the same curvature during the bonding process, the laminate losing some curvature and the actuator gaining curvature. This common curvature would initially be unknown. Step II of the model was developed to simulate this vacuum bonding technique, i.e., to determine the shape of the laminate-actuator combination resulting from the vacuum bonding process. This resulting shape of the laminate-actuator combination when the adhesive had cured will be referred to as the first actuator-added shape. With the actuator bonded to the laminate, there would be another unknown shape to which the laminate-actuator combination could be snapped. This other shape will be found in Step III, and will be referred to as the second actuator-added shape.

The total potential energy of the laminate and MFC actuator bonded to it in Step II was the sum of the strain energy from eq. $3, \Pi_{1}$, and the strain energy of the actuator, which is given as

$$
\Pi_{2}=4\left(\frac{1}{2} \int_{0}^{\frac{L_{x}^{M F C}}{2}} \int_{0}^{\frac{L_{y}^{M F C}}{2}} \int_{z_{2}}^{z_{3}}\left[\sigma_{x}^{a 2} \varepsilon_{x}^{a 2}+\sigma_{y}^{a 2} \varepsilon_{y}^{a 2}+\sigma_{x y}^{a 2} \gamma_{x y}^{a 2}\right] d x d y d z\right)
$$

where $\sigma_{x}^{a 2}, \sigma_{y}^{a 2}$, and $\sigma_{x y}^{a 2}$ and $\varepsilon_{x}^{a 2}, \varepsilon_{y}^{a 2}$, and $\gamma_{x y}^{a 2}$ are the stresses and strains in the MFC actuator due to the bonding process. As shown in fig. 1, the inplane dimensions of the actuator when flat are $L_{x}^{M F C}$ and $L_{y}^{M F C}$. The thickness coordinates $z_{2}$ and $z_{3}$ define the thickness of the actuator. Until the adhesive began to cure, the actuator could slip relative to the laminate, so the stresses created in the actuator due to the bonding process would be just bending stresses. The stress-strain relations for the bonded actuator are

$$
\begin{aligned}
& \sigma_{x}^{a 2}=\bar{Q}_{11} \varepsilon_{x}^{a 2}+\bar{Q}_{12} \varepsilon_{y}^{a 2}+\bar{Q}_{16} \gamma_{x y}^{a 2} \\
& \sigma_{y}^{a 2}=\bar{Q}_{12} \varepsilon_{x}^{a 2}+\bar{Q}_{22} \varepsilon_{y}^{a 2}+\bar{Q}_{26} \gamma_{x y}^{a 2} \\
& \sigma_{x y}^{a 2}=\bar{Q}_{16} \varepsilon_{x}^{a 2}+\bar{Q}_{26} \varepsilon_{y}^{a 2}+\bar{Q}_{66} \gamma_{x y}^{a 2}
\end{aligned}
$$


For simplification, it is assumed that the curvatures of the midsurface of the laminate and the midsurface of the actuator (rather than the curvatures of the contacting surfaces) are the same. The strains in the actuator may then be approximated as

$$
\begin{aligned}
& \varepsilon_{x}^{a 2}=\left(z-\left(\frac{z_{3}-z_{0}}{2}\right)\right) \kappa_{x}^{0} \\
& \varepsilon_{y}^{a 2}=\left(z-\left(\frac{z_{3}-z_{0}}{2}\right)\right) \kappa_{y}^{0} \\
& \gamma_{x y}^{a 2}=\left(z-\left(\frac{z_{3}-z_{0}}{2}\right)\right) \kappa_{x y}^{0}=0
\end{aligned}
$$

where the $\kappa \mathrm{s}$ are not known, but will be the same in the actuator and the laminate. The displacement fields of the form of eq. 2 are again assumed to be valid. For Step II, the total potential energy of the laminate-actuator combination is given as

$$
\Pi=\Pi_{1}+\Pi_{2}
$$

It should be noted in eq. 13 that, in the total potential energies of the laminate and actuator, the work terms due to the stresses between the laminate and actuator cancel each other. The coefficients $c_{1}$ through $c_{10}$ for this step are again determined by finding stationary values of $\Pi$, as in eq. 9 , and stability is checked by examining the second variation.

It is important to again delineate at this point the consequences of bonding the actuator to the laminate. The analysis shows that when cooled, the two-layer $[0 / 90]_{\mathrm{T}}$ cross-ply laminate considered could have either one stable equilibrium shape, or it could have an unstable saddle shape and two stable cylindrical shapes, depending on the dimensions of the laminate. In the latter case, the two cylindrical shapes would have equal and opposite radii of curvature and perpendicular generating axes. The actuator could be bonded to the cooled laminate when the laminate was in either of these two cylindrical shapes. As a result of bonding the actuator to the laminate with the laminate in a particular shape, that particular shape would change, and the shape to which it could be snapped to would also change. The shape resulting from Step II, the first actuator-added shape, would be the initial shape for Step III.

\section{Step III: Actuation of the MFC}

Step III of the model considered the laminate-actuator combination with no slipping between the laminate and the actuator, and included the deformation due to voltage applied to the actuator. The initial shape was to be specified by the coefficients $c_{1}$ through $c_{10}$ associated with the shape

determined from Step II; for Step III, these known coefficients were renamed $c_{1}^{i}$ through $c_{10}^{i}$, respectively. The superscript ' $i$ ' denotes 'initial.' The total potential energy of the model was again 
broken into the laminate contribution, again $\Pi_{1}$, and the actuator contribution, $\Pi_{3}$. The contribution to the total potential energy from the MFC actuator in Step III is written as

$$
\Pi_{3}=4\left(\frac{1}{2} \int_{0}^{\frac{L_{x}^{M F C}}{2}} \int_{0}^{L_{y}^{M F C}}{ }_{z_{2}}^{z_{3}}\left[\left(\sigma_{x}^{a 3}-\sigma_{x}^{E}\right) \varepsilon_{x}^{a 3}+\left(\sigma_{y}^{a 3}-\sigma_{y}^{E}\right) \varepsilon_{y}^{a 3}+\left(\sigma_{x y}^{a 3}-\sigma_{x y}^{E}\right) \gamma_{x y}^{a 3}\right] d x d y d z\right)
$$

where the $\varepsilon^{a 3} \mathrm{~s}$ and $\gamma_{x y}^{a 3}$ are the strains in the actuator and are to be discussed below. The midsurface of the graphite-epoxy laminate remains the reference surface. The $\sigma^{a 3} \mathrm{~s}$ are the stresses in the actuator and are given by

$$
\begin{aligned}
& \sigma_{x}^{a 3}=\bar{Q}_{11} \varepsilon_{x}^{a 3}+\bar{Q}_{12} \varepsilon_{y}^{a 3}+\bar{Q}_{16} \gamma_{x y}^{a 3}-\sigma_{x}^{E} \\
& \sigma_{y}^{a 3}=\bar{Q}_{12} \varepsilon_{x}^{a 3}+\bar{Q}_{22} \varepsilon_{y}^{a 3}+\bar{Q}_{26} \gamma_{x y}^{a 3}-\sigma_{y}^{E} \\
& \sigma_{x y}^{a 3}=\bar{Q}_{16} \varepsilon_{x}^{a 3}+\bar{Q}_{26} \varepsilon_{y}^{a 3}+\bar{Q}_{66} \gamma_{x y}^{a 3}-\sigma_{x y}^{E}
\end{aligned}
$$

The $\sigma^{E} \mathrm{~s}$ are piezoelectrically-induced stresses and are given by

$$
\begin{aligned}
& \sigma_{x}^{E}=\bar{Q}_{11} \varepsilon_{x}^{E}+\bar{Q}_{12} \varepsilon_{y}^{E}+\bar{Q}_{16} \gamma_{x y}^{E} \\
& \sigma_{y}^{E}=\bar{Q}_{12} \varepsilon_{x}^{E}+\bar{Q}_{22} \varepsilon_{y}^{E}+\bar{Q}_{26} \gamma_{x y}^{E} \\
& \sigma_{x y}^{E}=\bar{Q}_{16} \varepsilon_{x}^{E}+\bar{Q}_{26} \varepsilon_{y}^{E}+\bar{Q}_{66} \gamma_{x y}^{E}
\end{aligned}
$$

where the $\varepsilon^{E}$ S and $\gamma_{x y}^{E}$ are piezoelectrically-induced strains. The strains in the actuator, $\varepsilon^{a 3}$ s and $\gamma_{x y}^{a 3}$, are given by

$$
\begin{gathered}
\varepsilon_{x}^{a 3}=\varepsilon_{x}-\varepsilon_{x}^{s} \\
\varepsilon_{y}^{a 3}=\varepsilon_{y}-\varepsilon_{y}^{s} \\
\gamma_{x y}^{a 3}=\gamma_{x y}-\gamma_{x y}^{s}
\end{gathered}
$$

where the $\varepsilon s$ and $\gamma_{x y}$ are Kirchhoff-like strains that are zero at the reference surface (i.e., at the midplane of the laminate) and vary linearly with thickness. The $\varepsilon^{s}$ s and $\gamma_{x y}^{s}$ are considered shift strains and account for the discontinuous through-thickness distributions of the strains due to the room-temperature bonding of the actuator to the laminate, and allow the strain in the actuator to be defined by reference-surface strains and curvatures. To explain, after the actuator is bonded to the laminate, the strains in the laminate would be due to cooling and due to bending from adding the actuator. The strains in the actuator would be due only to bending. As a result, the strain distributions through the thickness of the laminate and actuator would not be continuous. When the laminateactuator combination is snapped to the second actuator-added shape, the laminate and actuator would both have extensional and bending strains; the strain distributions would continue to be discontinuous between the laminate and actuator. With actuation, it was assumed, in the spirit of the Kirchhoff 
hypothesis, that the strain increments in the laminate and actuator would be continuous through the thickness. As a result, the profiles of strains through the laminate and actuator would remain discontinuous. To account for this discontinuity, yet have one set of coefficients, i.e., $c_{1}$ through $c_{10}$, to define the strains within the laminate and the actuator due to the application of voltage, the shift of strain measures was necessary. The shift strains are thus given by

$$
\begin{gathered}
\varepsilon_{x}^{s}=\varepsilon_{x}^{i 0}+\left(\frac{z_{3}-z_{0}}{2}\right) \kappa_{x}^{i 0} \\
\varepsilon_{y}^{s}=\varepsilon_{y}^{i 0}+\left(\frac{z_{3}-z_{0}}{2}\right) \kappa_{y}^{i 0} \\
\gamma_{x y}^{s}=\gamma_{x y}^{i 0}+\left(\frac{z_{3}-z_{0}}{2}\right) \kappa_{x y}^{i 0}
\end{gathered}
$$

where the $\varepsilon^{i 0} \mathrm{~s}$ and $\gamma_{x y}^{i 0}$ are the initial strains and the $\kappa^{i 0} \mathrm{~s}$ are the initial curvatures, i.e., the strains and curvatures at the reference surface from Step II. These initial strains and curvatures are known.

The total potential energy for the actuation portion of the model is thus

$$
\Pi=\Pi_{1}+\Pi_{3}
$$

The shapes, actuated or unactuated, of the laminate-actuator combination are determined by solving for $c_{1}$ through $c_{10}$ by equating to zero the first variation of the total potential energy of eq. 19 . Stability is checked by examining at the second variation.

\section{COMPARISON OF MODEL WITH EXPERIMENTS}

For this study, a $[0 / 90]_{\mathrm{T}}$ laminate, fabricated from AS4/3502 graphite-epoxy prepreg, was cured flat and then cooled to room temperature. A parameter study using the developed analytical model was conducted to determine the dimensions of the laminate, i.e., $L_{x}$ and $L_{y}$, that could be snapped by the actuator. (The performance of the MFC actuator had been calibrated in a separate step using a cantilevered aluminum plate.) As determined by the parameter study, the laminate was cut to 150 by $150 \mathrm{~mm}$. The dimensions of the laminate and curvatures of the two cylindrical shapes were measured. An MFC actuator was centrally bonded to one side of the laminate to form the $\left[0 / 90 / 0^{\mathrm{MFC}}\right]_{\mathrm{T}}$ laminateactuator combination.

The measurements of the laminate affected the inputs to the model as follows: When the curvatures of the laminate were measured, the major curvatures of the two stable shapes were not of the same magnitude. It was assumed that uneven resin bleed during manufacture was responsible for the unequal curvatures. In the model, it was found that by increasing the thickness of one layer and decreasing the thickness of the other layer a corresponding amount, the slightly different curvatures could be predicted quite accurately. Since the total thickness of the laminate was a measured quantity, 
the assumption of the layers having an equal but opposite thickness change was justified. It was also found that, over time, the laminate lost some curvature, presumably due to moisture absorption. The $\Delta T$ used in the model, in eq. 6 , was reduced to account for this loss of curvature. The numerical values of the specific properties that were used in the analytical model are given in Table 1.

To demonstrate the snap-through event, actuation experiments were performed. The experimental setup consisted of a voltage supply, voltage amplifier, strain gage amplifier/conditioner, voltmeter, a LabVIEW data acquisition system on a personal computer, and the laminate-actuator combination with a strain gage bonded to the side opposite the actuator. The output from the strain gage was used to determine when the snap through event occurred. To perform the experiment, the laminate-actuator combination was snapped, by hand, to the second actuator-added shape, fig. 2a. Since the analyses of the model assumed the edges of the laminate-actuator combination were free of any specified force resultants or displacements, the laminate-actuator combination was suspended, like a pendulum, by the strain gage wire when conducting the snap-through experiments. The voltage was increased from zero until the laminate-actuator combination snapped to a shape similar to that of fig. $2 \mathrm{c}$; the voltage was then decreased to zero and the laminate-actuator combination assumed the shape given by fig. $2 \mathrm{c}$. This procedure was repeated six times with good repeatability and an average snapping voltage of $1695 \mathrm{~V}$.

With the adjustments to the layer thicknesses and $\Delta T$, which were discussed above, the model was used to predict the behavior of the laminate as the voltage was varied. The values of $c_{1}, c_{2}, c_{3}$, and $c_{4}$ were computed as a function of the voltage increase. These relationships are shown in fig. 3 , the solid lines represent stable equilibrium shapes and the dashed lines represent the unstable equilibrium shapes. Recall, from eq. 2, that these coefficients describe the out-of-plane displacement of the laminate-actuator combination. As shown in fig. 3, for a given voltage level, each of these coefficients has either three solutions, or one solution. At voltage levels below $1262 \mathrm{~V}$ there are three solutions. For voltages greater than $1262 \mathrm{~V}$, there is just one solution for each of the coefficients. Consider the laminate-actuator combination at zero applied voltage in the shape similar to fig. 2 a, i.e., $c_{1} \approx-1 \mathrm{~m}^{-1}$ and $c_{3} \approx+95 \mathrm{~m}^{-2}$, while $c_{2}$ and $c_{4}$ are both approximately zero. As the voltage is increased from zero, both $c_{1}$ and $c_{3}$ increase in magnitude, while $c_{2}$ and $c_{4}$ remain close to zero. At $1262 \mathrm{~V}$, the solution exhibits limit-point behavior, and a further increase in voltage results in the solution jumping to a configuration with a large curvature in the $y$-direction and little curvature in the $x$-direction, i.e., $c_{1} \approx 0, c_{2} \approx-5.5 \mathrm{~m}^{-1}, c_{3} \approx 0, c_{4} \approx-45 \mathrm{~m}^{-2}$. With a return of the voltage to zero, the room-temperature equilibrium shape similar to fig. $2 \mathrm{c}$ results.

The two predicted room-temperature shapes of the laminate-actuator combination are shown along with the actual shapes in fig. 2. Though this figure illustrates only a qualitative comparison, 
which is quite good, the quantitative comparison is good also. Considering the simplicity of the model, the predicted snapping voltage of $1262 \mathrm{~V}$ also compares fairly well with the snapping voltage measured in the experiments of $1695 \mathrm{~V}$.

\section{CONCLUDING REMARKS}

An interesting concept for morphing structures has been presented. Modeling and experimental work was considered, and fairly good agreement between the model and experiment was found. Though this initial effort was successful, there are a number of directions in which this work can proceed. The most obvious direction would be to add another actuator to the other side of the laminate. This second actuator could be used allow the laminate to self-reset, i.e., snap from one shape to another and back to the original shape using only voltage applied to the actuators. The number of actuators on one or both sides, actuator dimensions and shapes, and actuator location can be varied to extend and build upon the particular problem studied. Changing the scale of the concept, i.e., changing laminate sidelength dimensions and number of layers, is also worth pursuing. Laminates with fiber angles other 0 and $90^{\circ}$ could lead to shape changes that include twist curvature. In addition, more detailed analysis techniques, such as finite-element analysis, may be needed to capture some of more local effects that occur in this type of structure. Further details of the present effort are available in ref. 7.

\section{ACKNOWLEDGMENTS}

Mr. Robert Simonds, Prof. Dwight Viehland, and Dr. Jie-Fang Li of Virginia Tech are to be thanked for helping with experimental aspects of this study.

\section{REFERENCES}

1. Hyer, M. W. "The Room-Temperature Shapes of Four-Layer Unsymmetric Cross-Ply Laminates," $J$. Composite Materials, Vol. 16, 1982, pp. 318-340.

2. Hyer, M. W. "Calculations of the Room-Temperature Shapes of Unsymmetric Laminates," Journal of Composite Materials, Vol. 15, 1981, pp. 296-310.

3. Dano, M.-L. and Hyer, M.W., "Thermally-Induced Deformation Behavior of Unsymmetric Laminates," Int. J. Solids Structures, Vol. 35, 2002, pp. 319-327.

4. Hufenbach, W., Gude, M. "Analysis and Optimisation of Multistable Composites Under Residual Stresses," Composite Structures, Vol. 55, No. 16, pp. 2201-2207.

5. Wilkie, W. K., Bryant, R. G., High, J.W., Fox, R. L., Hellbaum, R. F., Jalink, A., Jr., Little, B. D., and Mirick, P. H. "Low-Cost Piezocomposite Actuator for Structural Control Applications," SPIE's 7th Annual International Symposium on Smart Structures and Materials, Newport Beach, CA, March 5-9, 2000.

6. Wolfram, S., The Mathematica Book, Wolfram-Media, Champaign-Urbana and Cambridge University Press, New York, 1999. 
7. Schultz, M. R. and M. W. Hyer, "Snap Through of Unsymmetric Cross-Ply Laminates Using Piezoceramic Actuators," J. Intelligent Material Systems and Structures, vol. 14, no. 12, 2004, pp. 795-814. 
Table 1 Material properties and other inputs to analytical model

\begin{tabular}{|c|c|c|c|}
\hline Property & $\begin{array}{l}\text { AS4/3502 } \\
\text { (Layer 1) }\end{array}$ & $\begin{array}{c}\text { AS4/3502 } \\
\text { (Layer 2) }\end{array}$ & $\begin{array}{l}\text { MFC Actuator } \\
\text { (active portion) }\end{array}$ \\
\hline$E_{1}(\mathrm{GPa})$ & 132.0 & 128.0 & 29 \\
\hline$E_{2}(\mathrm{GPa})$ & 9.798 & 9.608 & 18 \\
\hline$G_{12}(\mathrm{GPa})$ & 5.112 & 4.895 & 5.3 \\
\hline$v_{12}$ & 0.2990 & 0.3009 & 0.28 \\
\hline$\alpha_{1}\left(1 /{ }^{\circ} \mathrm{C}\right)$ & $-0.04156 \times 10^{-6}$ & $5.538 \times 10^{-9}$ & \\
\hline$\alpha_{2}\left(1 /{ }^{\circ} \mathrm{C}\right)$ & $23.77 \times 10^{-6}$ & $24.78 \times 10^{-6}$ & \\
\hline$d_{11}(\mu \varepsilon /(\mathrm{kV} / \mathrm{mm}))^{*}$ & & & 281 \\
\hline$d_{12}(\mu \varepsilon /(\mathrm{kV} / \mathrm{mm}))^{*}$ & & & -111 \\
\hline thickness (m) & $133.8 \times 10^{-6}$ & $138.2 \times 10^{-6}$ & $290 . \times 10^{-6}$ \\
\hline$L_{x}(\mathrm{~m})$ & \multicolumn{2}{|c|}{0.1518} & 0.08573 \\
\hline$L_{y}(\mathrm{~m})$ & \multicolumn{2}{|c|}{0.1518} & 0.05715 \\
\hline$\Delta x_{1}(\mathrm{~m})$ & & & $1.0668 \times 10^{-3}$ \\
\hline$\Delta T\left({ }^{\circ} \mathrm{C}\right)$ & \multicolumn{2}{|c|}{-117} & \\
\hline
\end{tabular}

*determined by calibration with cantilevered aluminum plate

\section{FIGURE CAPTIONS}

Fig. 1 Geometry of laminate-actuator combination: (a) cross-section view, (b) top view

Fig. 2 Experimental and predicted stable shapes of laminate-actuator combination with zero applied voltage

Fig. 3 Coefficients $c_{1}$ through $c_{4}$ Vs. applied voltage, showing existence of snap through 


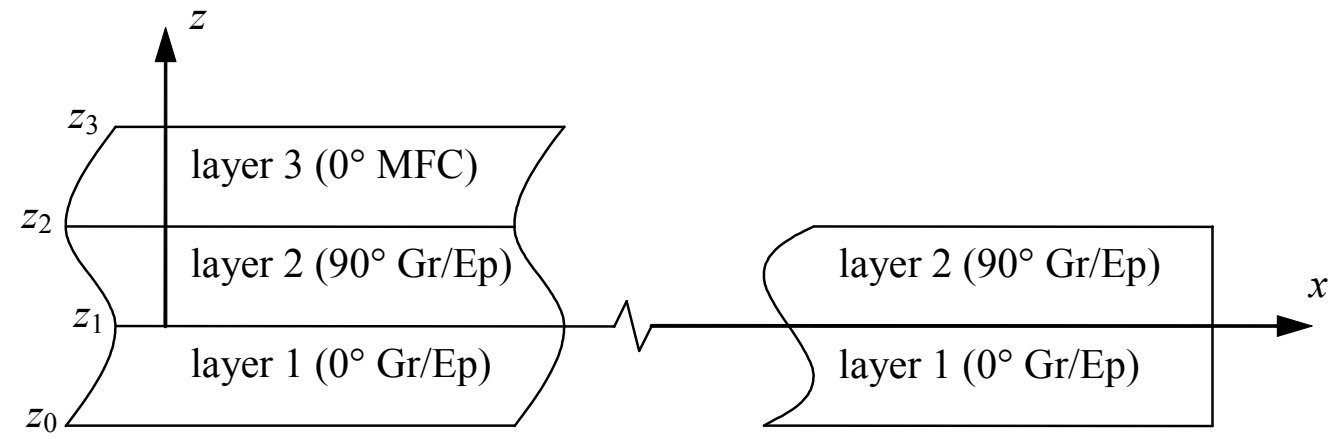

(a)

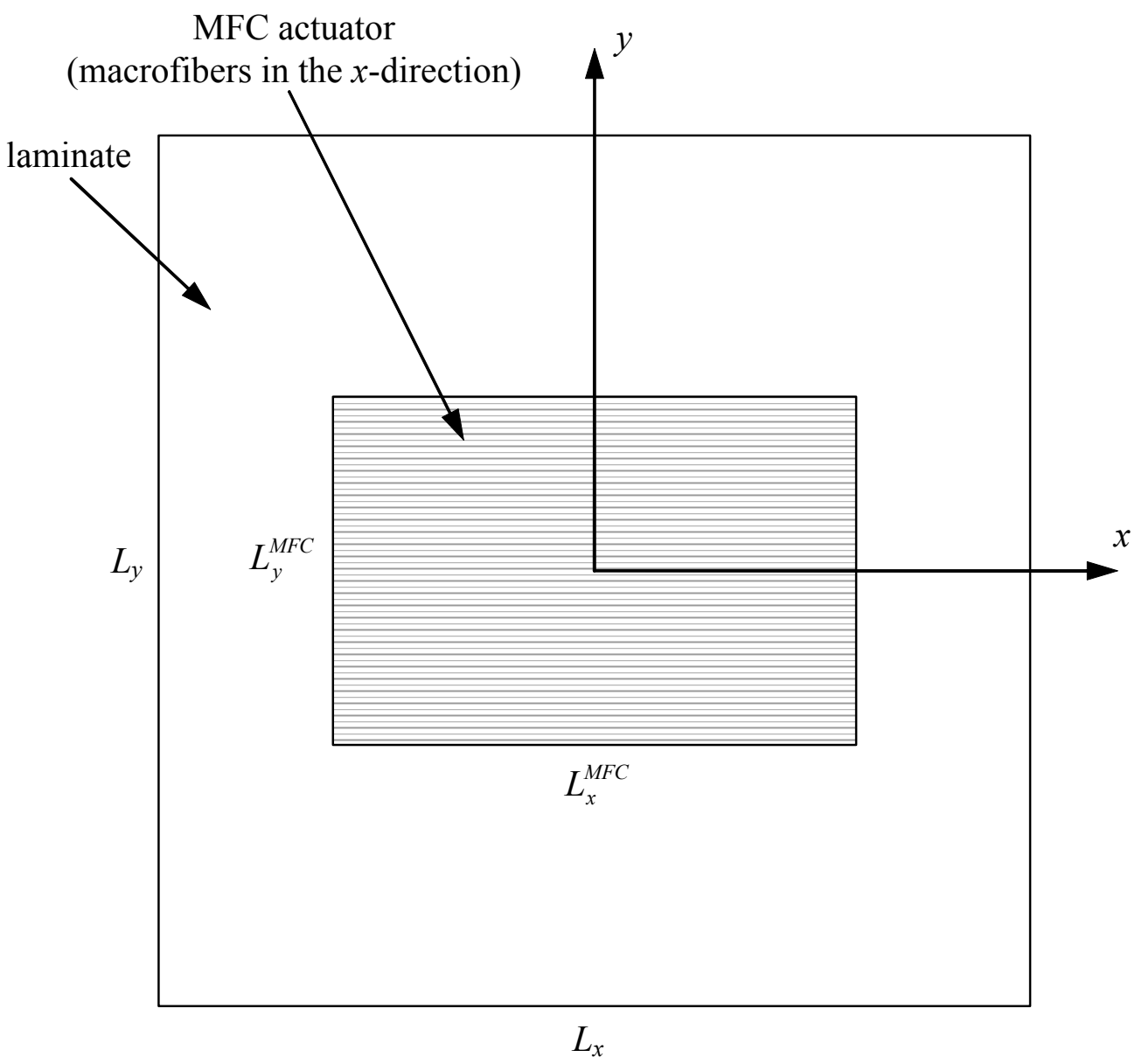

(b)

Fig. 1 Geometry of laminate-actuator combination: (a) cross-section view, (b) top view 
$\longrightarrow$

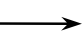

$\downarrow$

$\downarrow$ 

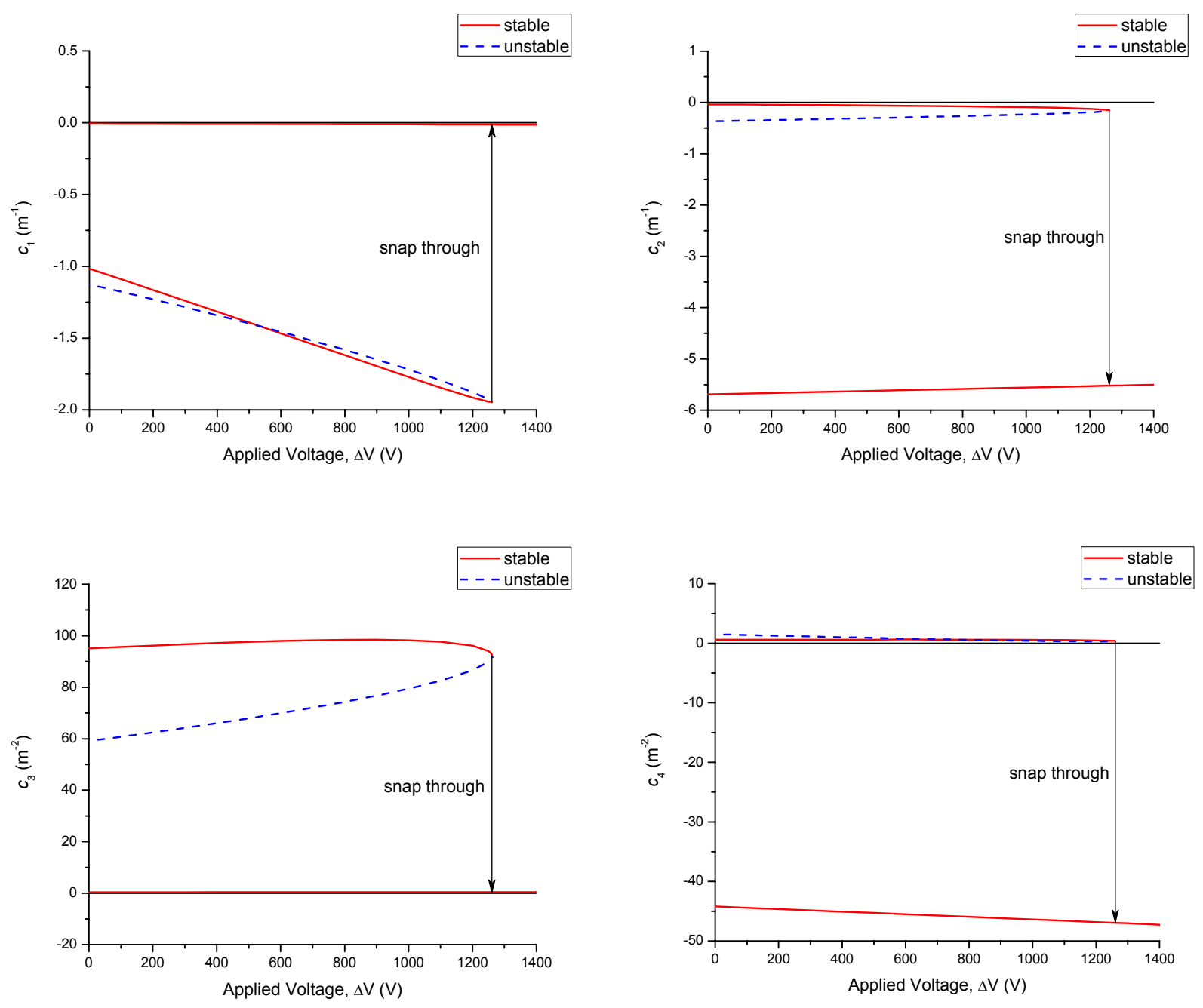

Fig. 3 Coefficients $c_{1}$ through $c_{4}$ vs. applied voltage, showing existence of snap through 